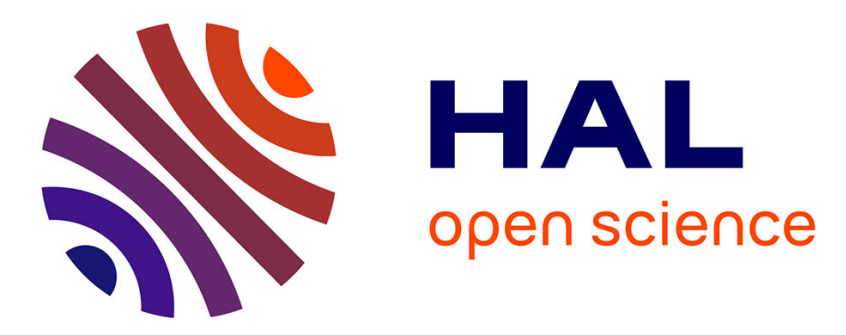

\title{
A model of scholarly publishing with hybrid academic journals
}

Damien Besancenot, Radu Vranceanu

\section{To cite this version:}

Damien Besancenot, Radu Vranceanu. A model of scholarly publishing with hybrid academic journals. 2014. hal-00971541

\section{HAL Id: hal-00971541 \\ https://essec.hal.science/hal-00971541}

Preprint submitted on 3 Apr 2014

HAL is a multi-disciplinary open access archive for the deposit and dissemination of scientific research documents, whether they are published or not. The documents may come from teaching and research institutions in France or abroad, or from public or private research centers.
L'archive ouverte pluridisciplinaire HAL, est destinée au dépôt et à la diffusion de documents scientifiques de niveau recherche, publiés ou non, émanant des établissements d'enseignement et de recherche français ou étrangers, des laboratoires publics ou privés. 


\section{A Model of Scholarly Publishing with Hybrid Academic Journals}

Research Center

ESSEC Working Paper 1406

2014

Damien Besancenot

Radu Vranceanu 
March 23, 2014

\title{
A MODEL OF SCHOLARLY PUBLISHING WITH HYBRID
}

\section{ACADEMIC JOURNALS}

\section{Damien Besancenot*and Radu Vranceanu ${ }^{\dagger}$}

\begin{abstract}
In April 2013, all of the major academic publishing houses moved thousands of journal titles to an original hybrid model, under which authors of accepted papers can choose between an expensive open access track and the traditional track available only to subscribers. This paper argues that authors might use publication strategy as a quality signaling device. The imperfect information game between authors and readers presents several types of Perfect Bayesian Equilibria, including a separating equilibrium in which only authors of high quality papers are driven toward the open access track. The publishing house will choose the open-access publication fee that supports the emergence of the highest return equilibrium. Journal structures will evolve over time according to the journals' accessibility - quality profiles.
\end{abstract}

Keywords: Academic publishing, Open access, Knowledge diffusion, Imperfect information, Signaling. JEL Classification: D52, L23, A14

\footnotetext{
*University of Paris 13 and CEPN, 99 rue Jean-Baptiste Clément, 93430, Villetaneuse, France. E-mail: besancenot.damien@univ-paris13.fr

${ }^{\dagger}$ ESSEC Business School and THEMA, 105 Av. Bernard Hirsch, 95021 Cergy, France. E-mail:
} vranceanu@essec.fr 


\section{Introduction}

Scholarly publishing of articles in peer-reviewed journals is by far the most important research communication channel. Depending on the underlying economic model, there are two well-established types of peer-reviewed journals: traditional and open access. Using an economic model established three centuries ago, a traditional journal restricts its access only to subscribers. With the advent of the Internet and the ubiquitous portable document format (pdf), traditional journals created their own websites and, gradually, users shifted from reading the printed edition to downloading and reading electronic versions of the journal provided that they were subscribers. Traditional journals will grant free access to a very small number of high impact papers as a teaser for marketing purposes. A small number of journals in Science allow for self-archiving, making the papers available for free.

At the same time, the Internet supported the emergence of an alternative economic model for scholarly publishing in so-called open access (OA) journals. OA journals maintain an editorial board and run a more or less demanding paper selection process. They allow web users to freely download papers; in turn, to cover the administrative costs and make a profit, many open access journal will charge a publication fee to the authors. In December 2013, the Directory of Open Access journals recorded 10.000 titles and this number is increasing steadily. ${ }^{1}$

Until very recently, a significant majority of leading journals relied on the traditional model. In the last twenty years the academic publishing business underwent a dramatic concentration process, driven by successive mergers between large players and waves of acquisition of smaller presses (Bowen et al. 2013). As a result, most academic journals are now owned by large size, forprofit publishing companies. In 2006 - Reed Elsevier, Springer and Taylor and Francis - accounted for $60 \%$ of all journal titles indexed in the ISI Web of Science. If we add the collections by Sage and John Wiley and Sons (who acquired Blackwell in 2007), it is clear that these five Major Publishing Houses (MPHs) have a tight grip on the traditional journal market.

With their increased market power, MPHs have the ability to implement more aggressive

\footnotetext{
${ }^{1}$ Data are available on the website of the Directory of Open Access journals at www.doaj.org.
} 
marketing strategies, such as journal bundling, which allows them to charge higher prices and reap higher profits (Edlin and Rubinfeld, 2005; Nevo et al. 2005; Dewatripoint et al. 2007). ${ }^{2}$ In the last two decades, subscriptions fees to traditional journals have risen dramatically, with a much steeper increase recorded by for-profit journals compared to non-profit ones. As a consequence, the affordability of these journals has gradually declined. Limiting access to knowledge to only those who can afford to pay a high price has a dubious ethical dimension and can harm long-term economic growth by setting a cap on human capital accumulation.

To avoid these charges while protecting their high return, in April 2013, the aforementioned big-5 MPHs operated a coordinated move to replace the traditional model with a "hybrid" or "two-track" academic journal model. All authors of accepted papers now have the choice between the "traditional" track and an "open access" track (of the same journal). The traditional track is free, but only readers who paid the journal subscription fee have access to the paper, either via the Internet or in the printed version of the journal. If the author chooses the open access track, he must pay a substantial publication fee, ranging between 500 to 5000 USD (most often more than 1500 USD). In turn, the paper becomes freely available to all Internet users. Hence, the composition of traditional journals might gradually evolve toward a hybrid structure, with a subset of papers published under the traditional, restricted access track, and a subset of papers published under the OA track. Because of the increased revenue flow from authors, the MPHs have already announced they will reduce the 2015 subscription fees for journals with a large number of OA papers. For instance, Taylor and Françis indicates clearly that if "paid OA content makes up $95 \%$ of a journal's content in a volume year, we will convert that title to pure OA in the following year". ${ }^{3}$

While hybrid journals existed before 2013, they were seen as a scant curiosity. As note by Walker (2004), the Entomological Society of America, which runs four important academic journals, was the first to sell Open Access by article in 2000, followed by the American Society of Limnology and Oceanography, who adopted the hybrid model in 2003. The early experience of

\footnotetext{
2 According to their financial statements, operating profit margins of MPHs are close to $30 \%$.

${ }^{3}$ See www.tandfonline.com/page/ openaccess/funders\#hybrid-pricing as accessed on March 1st, 2014.
} 
these small presses was positive, with a substantial increase in net revenues, and the proportion of authors that chose the OA track rose during the first years of adoption.

This paper aims to analyze the prospective evolution of scholarly publishing given the rise of hybrid academic journals. As an original contribution to the literature on scholarly publishing, it investigates whether authors can use the new publication options to signal the quality of their papers, taking into account readers' beliefs and the profit-maximization behavior of the publishing house. This research question is motivated by existing empirical analyses of the relationship between the number of citations, as a proxy for academic impact, and the recourse to OA facilities as provided by traditional journals. Early empirical analyses have shown that OA papers tend to be cited 3 to 6 times more than restricted access papers (e.g., Lawrence, 2001; Harnad and Brody, 2004). However, Kurtz et al. (2005), Gaulé and Maystre (2011) and McCabe and Snyder (2013a; 2013b) found that the high academic impact of OA papers is explained by their better intrinsic quality, and not the higher accessibility of the OA facilities. They conclude that the self-selection bias can explain most if not all of the positive impact of OA on citations as observed in the early studies.

The model is cast as an imperfect information game between authors of accepted papers, readers, and one profit-maximizing MPH which manages one representative hybrid journal. The MPH total revenue comprises publication fees collected on papers registered for the OA track, plus the subscription fee. Because the OA track provides a better visibility of their work, authors of high quality papers have an incentive to choose this track even if it means that they have to pay the publication fee. However, in this case authors of low quality papers might try to benefit from the positive signal and follow the same publication strategy. As all signaling games, this game presents several equilibria in pure and mixed strategies; it will be shown that the type of equilibrium depends on the OA publication fee. ${ }^{4}$ Hence the MPH can use the fee as an equilibrium selection device; it will choose the fee that maximizes its total revenue. This adds a screening level to the traditional signalling model used to analyze the behavior of authors and readers.

In this paper we assume that there is only one MPH, running one journal. Our approach

\footnotetext{
${ }^{4}$ We follow the classical methodology pioneered by Spence (1973).
} 
is justified since so far the competition among MPHs appears to be relatively weak. Should competition in the publication market increase in the future, the strategic interaction between MPHs must be taken into account. We also consider that the publication fee represents a cost for the author who can afford to pay for it. The degree of complexity of the problem would increase without changing fundamentally if we assume that some of the authors of the good papers cannot afford to pay the fee. Furthermore, should authors' employers decide to cover the publication fee in part or in full, a more sophisticated analysis would be required to account for the strategic behavior of the employer.

Given that the generalized adoption of the hybrid model by the MPHs is relatively recent, there are few theoretical papers to which we can directly relate our analysis. One notable set of studies includes analyses by McCabe and Snyder (2005; 2007) and Jeon and Rochet (2010) who modelled the academic publication market as a two-side platform with journals playing the mediation role between authors and readers. Each side of the platform - authors and readers - benefit from the positive externalities of the other side: authors enjoy a larger readership and readers like journals publishing many papers of a high quality. The quality of the papers depends on the resources spent for paper selection. Editors, who aim at maximizing profits, must arbitrate between a higher subscription fee (reducing the number of readers) and a higher OA publication fee (reducing the number of authors). Depending on the parameters of the problem, journals would opt for the traditional model, for the OA model, or for the hybrid structure. While these studies explicitly model the process of selecting top quality papers, they simplify the information structure of the problem, either by considering that authors do not know the quality of their paper or that the journal can detect this quality without error. Thus, they cannot study the signaling behavior by informed authors. At difference with them, our paper simplifies on the paper selection process by focusing on the accepted papers only, but builds on a more sophisticated, asymmetric information structure. This context allows us to address the signaling / screening problem as emphasized by the above mentioned empirical studies. ${ }^{5}$

\footnotetext{
5 The editorial selection process could be introduced along the lines of studies by Besancenot and Vranceanu (2008) or Besancenot et al. (2012).
} 
The rest of the paper is organized as follows. The first section introduces the main assumptions. Section 3 presents the equilibria of the game. In section 4, we determine the MPH's optimal pricing and preferred equilibrium. The last section concludes.

\section{Main assumptions}

The model features three types of agents: a publishing house that manages one hybrid journal, authors of accepted papers and readers.

The journal has accepted for publication a continuum of papers of mass one. Authors (of these accepted papers) know the quality of their paper. They chose a publication strategy such as to maximize the impact of their work.

Accepted papers are heterogenous with respect to their intrinsic quality. There are $\mu$ outstanding quality papers (with a quality index $\theta^{H}$ for "high quality") and $(1-\mu)$ standard quality papers (with a quality index $\theta^{L}$ for "low quality"), where $\theta^{H}>\theta^{L}$. Defining the quality of a paper is a challenging task. In a broad perspective, a high quality paper is probably both rigorous and relevant, i.e., its findings are useful to someone - peers in academia, decision makers or society at large, be it today, or in the years to come. To acknowledge that high quality research is a scarce good, we impose that $\mu<1 / 2$.

An author can choose to have his accepted paper published either under the traditional, restricted access track $(T-$ strategy $)$ or in the new, open access track $(A-$ strategy $)$. The frequency of authors of a high quality paper $\left(\theta^{H}\right)$ who play the $A-$ strategy is denoted by $\alpha$. The frequency of authors of a low quality paper $\left(\theta^{L}\right)$ who play $A$ is denoted by $\beta$. Figure 1 presents the author Decision Tree.

If the author opts for the open access track, he must pay an OA publication fee denoted by $c$. There is no publication fee for the traditional track.

At the outset of the game, the MPH must post the OA publication fee (per article) and the subscription fee; given the standard legal constraints of the publication market, it cannot renegotiate the fee after observing authors' strategy. The MPH chooses the pricing rule that 
maximizes its income. ${ }^{6}$

Readers have only an imperfect information about the quality of a paper. They update their beliefs about a paper quality after observing the publication strategy of the author and they will learn the quality after reading the paper. The two dotted lines in Figure 1 indicate that, out of the special case of a separating equilibrium, readers cannot unambiguously infer the true quality of the paper from observing the publication strategy.

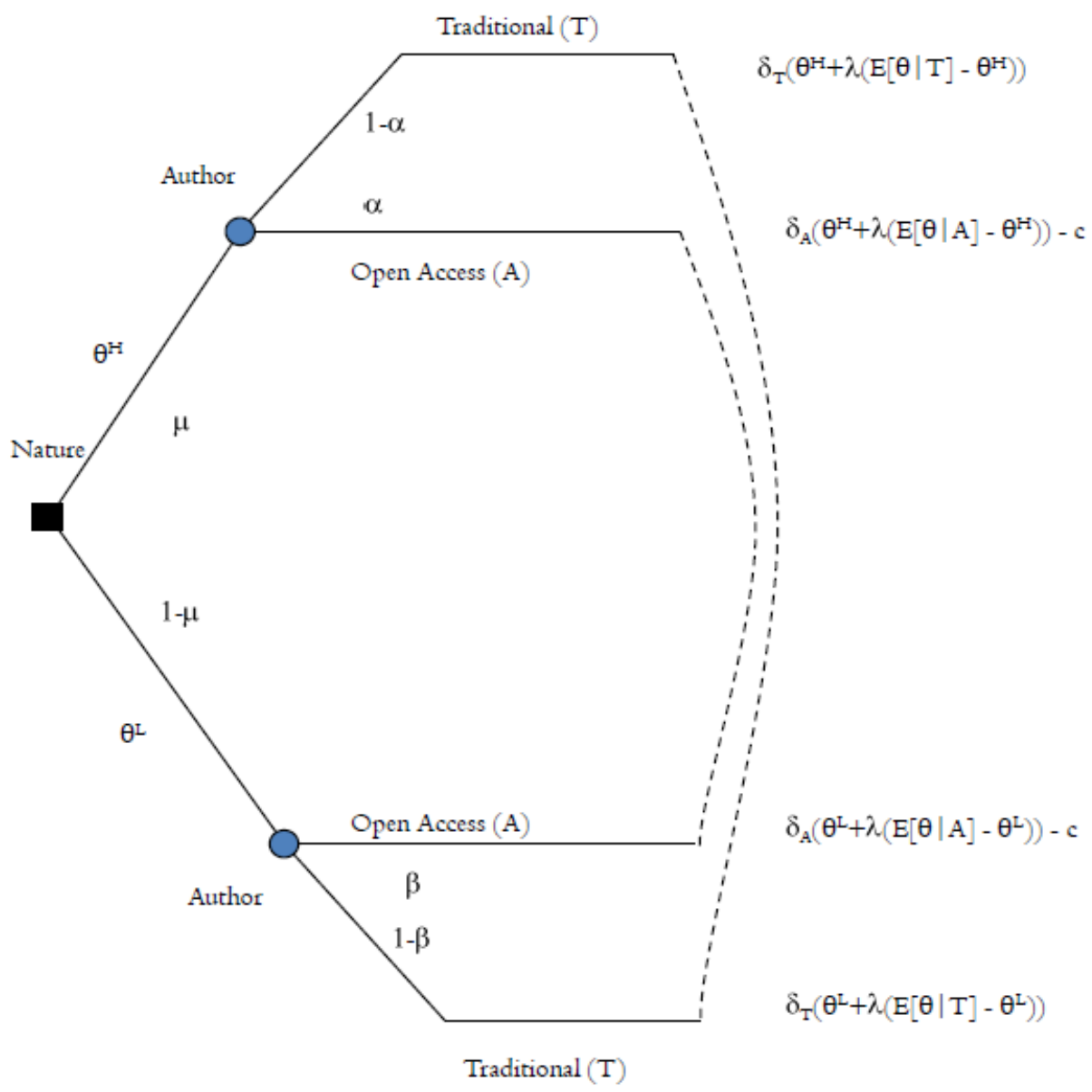

Figure 1: The Author's Decision Tree

\section{The author's payoff.}

We assume that the scholar's reward from a published paper depends on the impact of his work, that is the ability of his research to have a bearing on fellow scholars, decision makers or

\footnotetext{
${ }^{6}$ We implicitly assume that the cost of processing a paper does not vary from one track to another; we can set it to zero without a substantial loss in generality.
} 
society at large. Impact is assumed to depend on the paper's accessibility, and its intrinsic and perceived quality. More in detail, for a paper to have an impact, it must firstly reach a wide readership (Gaulé and Maystre, 2011). All things equal, potential readers have an easier access to papers published in the OA track as compared to papers published in the subscriber-only section of a journal. Next, high quality papers should have a larger social and academic impact than low quality papers just because they address a more important topic, with a better methodology. But since readers cannot gauge the quality of a paper before they read it, the impact of a paper also depends on their beliefs about the quality of the paper. For instance, if readers believe that a paper is of a high quality they will read it more carefully than if they believe the paper is of a low quality; thus, taking the same low quality paper, should the readers believe the paper is of high quality, the impact of the paper will be higher than if readers believe the quality is low (and vice-versa).

The former assumptions about the determinants of "impact" can be captured by a simple payoff function. We define the utility of an author of type $i \in\{H, L\}$ who plays strategy $S \in\{A, T\}$ as:

$$
U^{i}(S)=\delta_{S}\left[(1-\lambda) \theta^{i}+\lambda E[\theta \mid S]\right]-\mathbf{1}_{S} c
$$

The first term is the product between a measure of accessibility $\left(\delta_{S}\right)$ and a measure of quality. We assume that $\delta_{A}>\delta_{T}$ to acknowledge that the open access track facilitates readers' access to the paper as compared to the traditional track. The measure of quality weights intrinsic quality $\left(\theta^{i}\right)$ and perceived quality $(E[\theta \mid S])$, with parameter $\lambda \in[0,1]$ shifting the balance between the two terms. We assume that intrinsic quality contributes to impact more than perceived quality, i.e. $\lambda<0.5$. Notice that expression $(1-\lambda) \theta^{i}+\lambda E[\theta \mid S]$ can be written in the equivalent form: $\theta^{i}+\lambda\left(E[\theta \mid S]-\theta^{i}\right)$, where $\lambda$ grasps the "reputation effect". Finally, the indicator function $\mathbf{1}_{S}$ takes the value 1 if $S=A$ and 0 if else.

As already mentioned in the Introduction, one imperfect measure of a paper's impact is the number of citations it has received within a given period. Whether this accessibility effect is strong or weak, the topic is still open to debate. Using a sample of 100 journals in science and controlling for quality, McCabe and Snyder (2013b) found a net impact of OA publishing on citations of $8 \%$. 
It should be noticed that empirical studies use citations data from the ISI Web of Science, which covers only academic journals. In some fields, such as economics and management, researchers also seek to have a direct impact on practitioners and policymakers; this type of influence is probably not well captured by the number of citations in academic journals. In these special fields, the impact of OA can be much stronger. ${ }^{7}$

\section{The payoff of the MPH}

The MPH's income is made up of publication fees collected on OA articles, and the subscription fee.

The frequency of authors that choose the open access track is given by $\varphi=\mu \alpha+(1-\mu) \beta$. For every paper published in the open access track, the journal will collect a fee $c$ (and then the paper can be downloaded for free). The total amount collected as a publication fee is $\varphi c$.

What can be the amount of the subscription fee? Since the open access track is available for free for all Internet users, the subscription fee should be related to the number and quality of the papers available in the traditional track. In order to keep the analysis simple but realistic, we assume that the subscription fee is the product of the number of papers and their average quality, i.e.: $(1-\varphi) E[\theta \mid T]$.

Thus the payoff function of the MPH can be written as:

$$
R=\varphi c+(1-\varphi) E[\theta \mid T]
$$

It is important to remark that the only strategic decision of the MPH is the choice of the OA fee. As will be shown latter on, this variable determines the frequency $\varphi$ and the average quality of the papers available in the traditional track, $E[\theta \mid T]$.

\section{Equilibria of the game}

A Perfect Bayesian Equilibrium equilibrium of the sub-game between authors and readers is defined as a situation where authors choose their optimal publication strategy given readers' beliefs, and readers' beliefs are correct given the optimal strategy of the authors. Furthermore, the MPH

\footnotetext{
7 The number of citations reported by Google Scholar, a database that covers a much broader range of publication outlets, would provide a better measure of such "societal" impact.
} 
chooses the publication fee such as to maximize its revenue, given the equilibrium strategies of authors and readers.

\subsection{Conditional probabilities and authors' payoffs}

Before solving for the equilibria of the game between readers and authors, we use Bayes rule to determine the conditional probability that a paper has a high quality given the publication strategy $S \in\{T, A\}:$

$$
\begin{aligned}
\operatorname{Pr}\left[\theta^{H} \mid A\right] & =\frac{\operatorname{Pr}\left[A \mid \theta^{H}\right] \operatorname{Pr}\left[\theta^{H}\right]}{\operatorname{Pr}\left[A \mid \theta^{H}\right] \operatorname{Pr}\left[\theta^{H}\right]+\operatorname{Pr}\left[A \mid \theta^{L}\right] \operatorname{Pr}\left[\theta^{L}\right]}=\frac{\alpha \mu}{\mu \alpha+\beta(1-\mu)} \\
\operatorname{Pr}\left[\theta^{H} \mid T\right] & =\frac{\operatorname{Pr}\left[T \mid \theta^{H}\right] \operatorname{Pr}\left[\theta^{H}\right]}{\operatorname{Pr}\left[T \mid \theta^{H}\right] \operatorname{Pr}\left[\theta^{H}\right]+\operatorname{Pr}\left[T \mid \theta^{L}\right] \operatorname{Pr}\left[\theta^{L}\right]}=\frac{(1-\alpha) \mu}{(1-\alpha) \mu+(1-\beta)(1-\mu)}
\end{aligned}
$$

Thus, the expected quality of a paper given $S \in\{T, A\}$ is :

$$
\begin{aligned}
& E[\theta \mid A]=\frac{\alpha \mu \theta^{H}+(1-\mu) \beta \theta^{L}}{\mu \alpha+(1-\mu) \beta} \\
& E[\theta \mid T]=\frac{(1-\alpha) \mu \theta^{H}+(1-\beta)(1-\mu) \theta^{L}}{(1-\alpha) \mu+(1-\beta)(1-\mu)}
\end{aligned}
$$

Introducing the expected quality in the general payoff definition (Eq. 1), after some calculations we obtain the payoffs for each type of author and each strategy:

$$
\begin{aligned}
U^{H}(A) & =\delta_{A}\left[\theta^{H}-\lambda \frac{(1-\mu) \beta\left(\theta^{H}-\theta^{L}\right)}{\mu \alpha+(1-\mu) \beta}\right]-c \\
U^{H}(T) & =\delta_{T}\left[\theta^{H}-\lambda \frac{(1-\mu)(1-\beta)\left(\theta^{H}-\theta^{L}\right)}{(1-\alpha) \mu+(1-\beta)(1-\mu)}\right] \\
U^{L}(A) & =\delta_{A}\left[\theta^{L}+\lambda \frac{\alpha \mu\left(\theta^{H}-\theta^{L}\right)}{\mu \alpha+(1-\mu) \beta}\right]-c \\
U^{L}(T) & =\delta_{T}\left[\theta^{L}+\lambda \frac{(1-\alpha) \mu\left(\theta^{H}-\theta^{L}\right)}{(1-\alpha) \mu+(1-\beta)(1-\mu)}\right]
\end{aligned}
$$

We can study now the various equilibria of this game.

\subsection{Separating equilibrium}

We study firstly the existence of a separating equilibrium where all authors of high quality papers play the $A$-strategy and all authors of standard quality papers adopt the traditional $T$-strategy. ${ }^{8}$ 
The equilibrium frequencies are $\alpha=1, \beta=0$ and conditional expectations (as defined here above) become: $E[\theta \mid A]=\theta^{H}$ and $E[\theta \mid T]=\theta^{L}$.

Thus the equilibrium requires two necessary conditions:

$$
\begin{gathered}
U^{H}(A)>U^{H}(T) \Leftrightarrow c<c_{1}=\delta_{A} \theta^{H}-\delta_{T}\left[\theta^{H}-\lambda\left(\theta^{H}-\theta^{L}\right)\right] \\
U^{L}(T)>U^{L}(A) \Leftrightarrow c>c_{2}=\delta_{A}\left[\theta^{L}+\lambda\left(\theta^{H}-\theta^{L}\right)\right]-\delta_{T} \theta^{L} .
\end{gathered}
$$

It can be easily checked that $\delta_{A}>\delta_{T} \Rightarrow c_{2}<c_{1}$. The equilibrium exists if $c \in\left[c_{2}, c_{1}\right]$.

Given that $\varphi=\mu$ and $E[\theta \mid T]=\theta^{L}$, in this equilibrium the MPH payoff can be written as a function of $c$ :

$$
R_{\text {sep }}(c)=c \mu+(1-\mu) \theta^{L}
$$

\subsection{Pooling Equilibrium $A$}

We study the existence of a pooling equilibrium where all authors choose the OA track. In this case, $\alpha=1, \beta=1$. If readers are rational, equilibrium beliefs must respect $\operatorname{Pr}\left[\theta^{H} \mid A\right]=\mu$; we further assume that should one author decide to deviate from the equilibrium strategy and play the $T$ strategy (refuse to pay the open access fee), readers will consider that the paper has a low quality: $\operatorname{Pr}\left[\theta^{H} \mid T\right]=0$. With this system of beliefs, the expected quality of the papers is: $E[\theta \mid A]=\mu \theta^{H}+(1-\mu) \theta^{L}$ and $E[\theta \mid T]=\theta^{L}$.

For this equilibrium to occur, both types of authors must prefer the $A$ - strategy to the $T-$ strategy. This requires two following necessary conditions:

$$
\begin{aligned}
U^{H}(A) & >U^{H}(T) \Leftrightarrow c<\delta_{A}\left[\theta^{H}-\lambda(1-\mu)\left(\theta^{H}-\theta^{L}\right)\right]-\delta_{T}\left[\theta^{H}+\lambda\left(\theta^{L}-\theta^{H}\right)\right] \\
U^{L}(A) & >U^{L}(T) \Leftrightarrow c<\delta_{A}\left[\theta^{L}+\mu \lambda\left(\theta^{H}-\theta^{L}\right)\right]-\delta_{T} \theta^{L} .
\end{aligned}
$$

We can easily check that the first condition implies the second one. Thus the necessary condition for this equilibrium to exist is:

$$
c<c_{3}=\delta_{A}\left[\theta^{L}+\mu \lambda\left(\theta^{H}-\theta^{L}\right)\right]-\delta_{T} \theta^{L}
$$

\footnotetext{
${ }^{8}$ It can be shown that the opposite separating equilibrium does not exist.
} 
Given that all authors publish in the open access track $(\varphi=1)$ in this equilibrium the MPH payoff function is

$$
R_{P A}(c)=c .
$$

\subsection{Pooling Equilibrium $T$}

In the opposite pooling equilibrium all authors would choose the traditional track, that is $\alpha=0$, $\beta=0$. A rational reader would consider that $\operatorname{Pr}\left[\theta^{H} \mid T\right]=\mu$ and $\operatorname{Pr}\left[\theta^{H} \mid A\right]=1-\mu$. We further assume that an author who deviate and choose the A-strategy (pays the fee) would be perceived as the author of a high quality paper: $\operatorname{Pr}\left[\theta^{H} \mid A\right]=1$. According to this system of beliefs, the expected quality of the papers are $E[\theta \mid T]=\mu \theta^{H}+(1-\mu) \theta^{L}$ and $E[\theta \mid A]=\theta^{H}$. This equilibrium exists if authors prefer the traditional track whatever the quality of their paper:

$$
\begin{aligned}
& U^{H}(A)<U^{H}(T) \Leftrightarrow c>\delta_{A} \theta^{H}-\delta_{T}\left[\theta^{H}-\lambda(1-\mu)\left(\theta^{H}-\theta^{L}\right)\right] \\
& U^{L}(A)<U^{L}(T) \Leftrightarrow c>\delta_{A}\left[\theta^{L}+\lambda\left(\theta^{H}-\theta^{L}\right)\right]-\delta_{T}\left[\theta^{L}+\lambda \mu\left(\theta^{H}-\theta^{L}\right)\right] .
\end{aligned}
$$

It can be checked that the first condition implies the second one. Thus the necessary condition for this equilibrium to exist is:

$$
c>c_{4}=\delta_{A} \theta^{H}-\delta_{T}\left[\theta^{H}-\lambda(1-\mu)\left(\theta^{H}-\theta^{L}\right)\right] .
$$

In this equilibrium, the MPH payoff is a constant (it is independent of the publication fee):

$$
R_{P T}=E[\theta \mid T]=\mu \theta^{H}+(1-\mu) \theta^{L} .
$$

\subsection{Hybrid Equilibrium 1}

We consider the equilibrium where all authors of high quality papers play A $(\alpha=1)$, the others randomize between the open access and the traditional strategy $(0<\beta<1)$. Given that the traditional strategy signals that the paper is of a low quality, it will never be played by the author of a high quality paper. The open access strategy can be adopted by both types. The expected quality is $E[\theta \mid T]=\theta^{L}$ and $E[\theta \mid A]=\frac{\mu \theta^{H}+(1-\mu) \beta \theta^{L}}{\mu+(1-\mu) \beta}$.

There are two equilibrium conditions. It can be shown that $U^{H}(A)>U^{H}(T)$ is always true. The second one states that author of a low quality paper should be indifferent between playing 
the $A$ and $T$ - strategy: $U^{L}(A)=U^{L}(T)$. This allows us to determine the equilibrium frequency of authors of low quality papers that will register for the open access track:

$$
\beta=\frac{\mu}{1-\mu}\left[\frac{\left(\theta^{H}-\theta^{L}\right)}{c-\left(\delta_{A}-\delta_{T}\right) \theta^{L}} \lambda \delta_{A}-1\right] .
$$

It turns out that $\beta=0$ for $c=c_{2}$ and $\beta=1$ for $c=c_{3}$ as defined by Equations (12) and (16). It can be also be observed that $c_{3}<c_{2}$. Thus this hybrid equilibrium exists for $c \in\left[c_{3}, c_{2}\right]$. Furthermore, in this equilibrium $d \beta / d c<0$ : the proportion of authors of low quality papers who adopt the Open Access strategy is declining with the open access fee.

The MPH payoff is:

$$
\begin{aligned}
R_{H 1}(c) & =[\mu+(1-\mu) \beta] c+[(1-\mu)(1-\beta)] \theta^{L} \\
& =\theta^{L}-\mu \lambda \delta_{A}\left(\theta^{H}-\theta^{L}\right)\left[\frac{\left(\theta^{L}-c\right)}{c-\left(\delta_{A}-\delta_{T}\right) \theta^{L}}\right] .
\end{aligned}
$$

This gain depends on $c$ as indicated by the derivative

$$
\frac{d R_{H 1}}{d c}=\lambda \delta_{A} \mu\left(\theta^{H}-\theta^{L}\right) \frac{\theta^{L}\left[1-\left(\delta_{A}-\delta_{T}\right)\right]}{\left[c-\left(\delta_{A}-\delta_{T}\right) \theta^{L}\right]^{2}}
$$

For $c \in\left[c_{3}, c_{2}\right]$, there are two distinct cases:

If $\delta_{A}>1+\delta_{T}$ then $d R_{H 1} / d c<0$. In this case, the MPH makes the highest profit if $c$ is as low as $c_{3}$; but at $c_{3}$ the hybrid equilibrium is nothing else than the Pooling Equilibrium $\mathrm{A}$, and the payoff of the MPH is the payoff in the Pooling Equilibrium A as evaluated for $c=c_{3}$.

If $\delta_{T}<\delta_{A}<1+\delta_{T}$, then $d R_{H 1} / d c>0$. In this case, the MPH should better push the fee up to $c_{2}$; but for $c=c 2$, the Hybrid equilibrium takes the extreme profile of the Separating equilibrium, and the MPH payoff is the same one obtained in the Separating Equilibrium as evaluated for $c=c_{2}$.

Thus, if the MPH aims at maximizing its payoff, it will never aim to create the conditions of existence of the Hybrid equilibrium 1 by choosing $c \in] c_{3}, c_{2}[$. We can thus eliminate the Hybrid equilibrium 1 from the subsequent analysis of the income maximization decision of the MPH.

\subsection{Hybrid Equilibrium 2}

In this case, all authors of low quality papers adopt the $T$ - strategy $(\beta=0)$ and authors of high quality papers randomize between the traditional and the open access track; $0<\alpha<1$. Since 
only high quality papers are presented in open access, this publication strategy reveals the quality of the paper. The traditional publication strategy can be adopted by both authors of high and low quality papers. The expected quality is $E[\theta \mid A]=\theta^{H}$ and $E[\theta \mid T]=\frac{(1-\alpha) \mu \theta^{H}+(1-\mu) \theta^{L}}{(1-\alpha) \mu+(1-\mu)}$.

There are two equilibrium conditions. The first $U^{L}(A)<U^{L}(T)$ is fulfilled whatever the parameter values. The second indifference condition $U^{H}(A)=U^{H}(T)$ defines the equilibrium proportion of high quality papers that is presented to the open access track:

$$
\alpha=1-\frac{(1-\mu)}{\mu}\left[\frac{\lambda \delta_{T}\left(\theta^{H}-\theta^{L}\right)}{c-\left(\delta_{A}-\delta_{T}\right) \theta^{H}}-1\right] .
$$

We can check that for $c=c_{4}$ we get $\alpha=0$, and for $c=c_{1}$ we get $\alpha=1$ with the two cutoff costs as defined by Equations (11) and (20). It can also be noticed that $c_{4}<c_{1}$. Thus, this hybrid equilibrium is feasible for $c \in\left[c_{4}, c_{1}\right]$.

Furthermore, it turns out that $d \alpha / d c>0$ : The proportion of authors of high quality papers who go for the open access track is increasing in the OA publication fee. ${ }^{9}$

In order to study the MPH income maximization problem, we firstly determine $E[\theta \mid T]$ replacing $\alpha$ by its expression in Eq. (25):

$$
E[\theta \mid T]=\frac{(1-\alpha) \mu \theta^{H}+(1-\mu) \theta^{L}}{(1-\alpha) \mu+(1-\mu)}=\theta^{H}-\frac{c-\left[\delta_{A}-\delta_{T}\right] \theta^{H}}{\lambda \delta_{T}} .
$$

The MPH revenue is:

$$
R_{H 2}(c)=\varphi c+(1-\varphi) E[\theta \mid T]=c+(1-\mu)\left(\theta^{H}-\theta^{L}\right)\left[\frac{\lambda \delta_{T}\left(\theta^{H}-c\right)}{c-\left(\delta_{A}-\delta_{T}\right) \theta^{H}}-1\right] .
$$

It can be shown that, depending on parameters, this revenue is monotonically increasing or decreasing with respect to $c$ :

$$
\frac{d R_{H 2}}{d c}=1+\lambda \delta_{T}(1-\mu)\left(\theta^{H}-\theta^{L}\right) \theta^{H} \frac{\left(\delta_{A}-\delta_{T}\right)-1}{\left[c-\left(\delta_{A}-\delta_{T}\right) \theta^{H}\right]^{2}}
$$

There is no internal solution to the revenue maximization problem in the Hybrid equilibrium 2. Should the market settle to this equilibrium, the MPH would either try to obtain the Separating equilibrium, by pushing the fee up to $c_{1}$, or would seek to obtain the Pooling Equilibrium $\mathrm{T}$ by

\footnotetext{
9 This property points out that this hybrid equilibrium is unstable: any minor expectation shock would push authors of high quality papers to shift massively either to the $T$ - strategy or to the A-strategy.
} 
decreasing the fee to $c_{4}$, and obtain the (constant) revenue $R_{P T}$ as defined in Eq.(21). In the subsequent analysis of the optimal decision of the MPH, we can thus discard any payoff specific to the Hybrid equilibrium 2 and focus on the highest payoff obtained in the pure strategy equilibria.

Finally, it can be shown that an equilibrium where both type of authors randomize between the open access and the traditional track is impossible; conditions $U^{H}(A)=U^{H}(T)$ and $U^{L}(A)=$ $U^{L}(T)$ cannot be fulfilled simultaneously.

\section{The MPH optimal pricing policy}

\subsection{The OA publication fee and the equilibrium existence}

As already mentioned, the publishing house uses the OA publication fee $(c)$ as a screening device; more precisely, it will tune the fee such as to create the conditions to obtain the type of equilibrium entailing the highest payoff. Recall that the payoff comprises the journal subscription and the collected OA fees.

Given that the equilibrium of the game depends on the OA publication fee, in order to solve the MPH payoff maximization problem we need (1) to see what equilibria are feasible when $c$ takes various positive values, and then (2) to compare the maximum payoffs across the feasible equilibria. In the previous section we have shown that the set of "relevant" equilibria comprises the Separating Equilibrium and the two Pooling Equilibria. Indeed, the two Hybrid equilibria are payoff-dominated by the Pooling Equilibrium T or the Separating Equilibrium.

We have also defined four threshold values of the OA fee, delineating the boundaries between equilibria:

$$
\begin{aligned}
& c_{1}=\delta_{A} \theta^{H}-\delta_{T}\left[\theta^{H}-\lambda\left(\theta^{H}-\theta^{L}\right)\right] \\
& c_{2}=\delta_{A}\left[\theta^{L}+\lambda\left(\theta^{H}-\theta^{L}\right)\right]-\delta_{T} \theta^{L} \\
& c_{3}=\delta_{A}\left[\theta^{L}+\mu \lambda\left(\theta^{H}-\theta^{L}\right)\right]-\delta_{T} \theta^{L} \\
& c_{4}=\delta_{A} \theta^{H}-\delta_{T}\left[\theta^{H}-\lambda(1-\mu)\left(\theta^{H}-\theta^{L}\right)\right]
\end{aligned}
$$

We have already checked that for $\mu<1$ and $\delta_{A}>\delta_{T}$, the following inequalities always hold: $c_{1}>c_{2}$ (see Separating equilibrium), $c_{2}>c_{3}$ (see Hybrid equilibrium 1) and $c_{4}<c_{1}$ (see Hybrid 
equilibrium 2). Moreover, we can show that $c_{3}<c_{4}$ (for $\mu<1 / 2$ ). Thus the thresholds can be ranked as:

$$
c_{3}<\left\{c_{2}, c_{4}\right\}<c_{1} .
$$

It can be shown that $c_{4}>c_{2}$ if $\delta_{A}>\delta_{T}\left[1+\mu \frac{\lambda}{(1-\lambda)}\right]$ and $c_{4}<c_{2}$ if $\delta_{T}<\delta_{A}<\delta_{T}\left[1+\mu \frac{\lambda}{(1-\lambda)}\right] \cdot{ }^{10}$

Figure 2 displays the equilibrium frequencies $\alpha$ and $\beta$ as a function of the $\mathrm{OA}$ fee, in the case where $c_{4}>c_{2}$. For a $\mathrm{OA}$ fee $0<c<c_{3}$, only the Pooling Equilibrium A would prevail ( $\alpha=1, \beta=1$ ); in this equilibrium, the MPH would maximize its payoff by choosing $c=c_{3}$. For $c_{3} \leq c \leq c_{2}$ Hybrid equilibrium 1 would prevail $(\alpha=1, \beta \in[0,1])$; yet we have shown that the MPH obtains the highest income at the extremes of the interval, that is either obtaining the payoff of the Pooling Equilibrium A or the Separating Equilibrium. For $c_{2}<c<c_{4}$ the Separating equilibrium is obtained $(\alpha=1, \beta=0)$. For $c_{4} \leq c \leq c_{1}$ the game presents a typical situation of multiple equilibria: any of the Separating, Hybrid 2 of Pooling T equilibria can exist. Which one will actually materialize depends on readers' beliefs and how the MPH has managed the OA fee. We have shown that in the Hybrid equilibrium 2, the MPH would prefer a fee at the boundaries of the interval; the actual choice is between $c_{1}$ implementing the Separating equilibrium, or $c_{4}$ implementing the Pooling Equilibrium T. If the Separating equilibrium brings the highest income, the MPH can make sure that readers' beliefs support this equilibrium by setting firstly a fee lower than $c_{4}$, then rising it gradually to $c_{1}$. Finally, by setting the OA fee above $c_{1}$ the MPH is sure to obtain the Pooling Equilibrium T and its constant subscription fee.

So in the Pooling Equilibrium A the MPH obtains the maximum payoff if it pushes the OA fee up to $c_{3}$. According to Eq. (17) this highest payoff is:

$$
R_{P A}\left(c_{3}\right)=\delta_{A}\left[\theta^{L}+\mu \lambda\left(\theta^{H}-\theta^{L}\right)\right]-\delta_{T} \theta^{L}
$$

According to Eq.(13), in the Separating Equilibrium the MPH gets the highest payoff if it brings the OA fee up to $c_{1}$. Its expression is:

$$
R_{S e p}\left(c_{1}\right)=c_{1} \mu+(1-\mu) \theta^{L}=\mu\left(\delta_{A} \theta^{H}-\delta_{T}\left[\theta^{H}-\lambda\left(\theta^{H}-\theta^{L}\right)\right]\right)+(1-\mu) \theta^{L} .
$$

\footnotetext{
${ }^{10}$ Given that $\lambda /(1-\lambda)<1$, a sufficient condition for $c_{4}>c_{2}$ is $\delta_{A}>\delta_{T}(1+\mu)$. Thus chances that $c_{4}>c_{2}$ are high if the proportion of outstanding papers $(\mu)$ is small.
} 


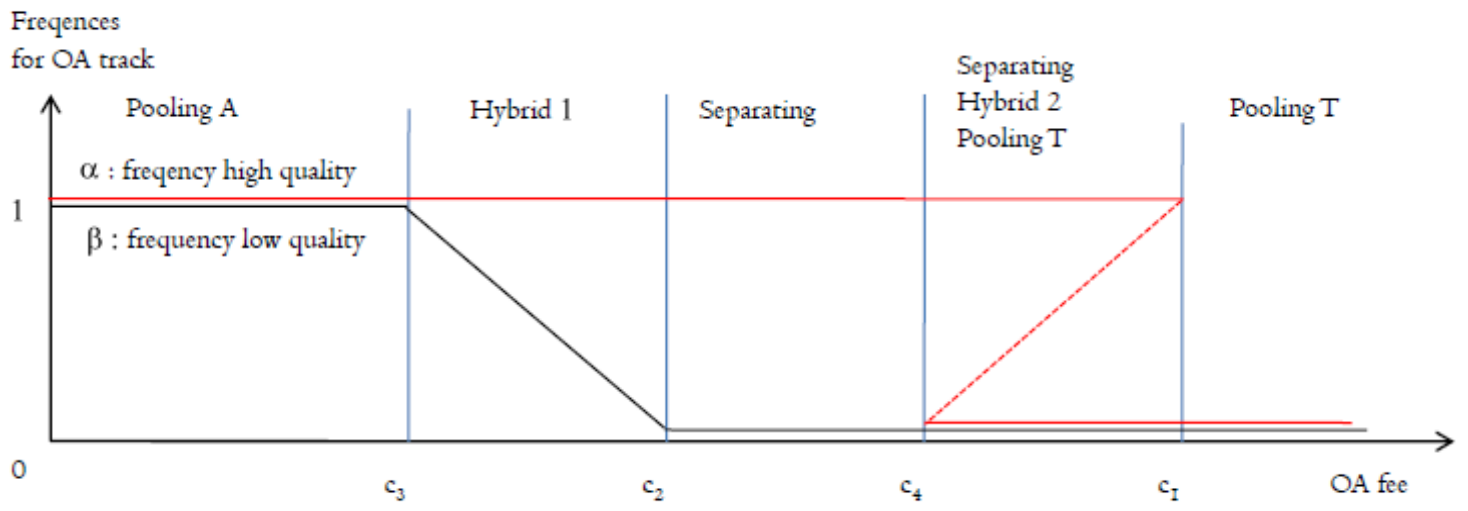

Figure 2: Equilibrium frequencies $\alpha$ and $\beta$

Finally, the highest payoff the MPH can get in the Pooling Equilibrium $\mathrm{T}$ is $R_{P T}$ as defined in equation (21). To obtain it, the MPH should push the OA fee above $c_{1}$, in which case no author will resort to the OA facility, and the journal can ask for the maximum subscription fee:

$$
R_{P T}=\mu \theta^{H}+(1-\mu) \theta^{L} .
$$

Notice that in the opposite case where $c_{4}<c_{2}$ the region where the Hybrid equilibrium 2 can exist would overlap with the separating equilibrium, but this does not modifies the decision problem of the MPH, nor the optimal fees. For the sake or parsimony we do not display this graph.

\subsection{The MPH preferred equilibrium}

In order to determine the global optimum, we need to compare two by two the highest payoff in each equilibrium as defined in Equations (29), (30), (31). Of course, the payoff ordering depends on all the parameters of the problem. In order to guide intuition, we need to restrict the parameter space to a tractable number. We thus choose to emphasize the quality gap and the accessibility gap. By quality gap we understand the relative position of $\theta^{H}$ with respect to $\theta^{L}$. The accessibility gap refers to the relative position of $\delta_{A}$ with respect to $\delta_{T}$.

Table 2 presents the payoff dominance conditions as imposed on accessibility; we must distinguish between the case of high quality gap and a low quality gap. The payoff inequalities depend on the position of $\delta_{A}$ relative to a term that is a linear function in $\delta_{T}$; these functions will be represented graphically as the $A A^{\prime}, B B^{\prime}$ and $C C^{\prime}$ lines (as denoted in the last column of Table 
2).

\begin{tabular}{|l|l|l|l|}
\hline \multicolumn{1}{|c|}{$\begin{array}{l}\text { MPH preferred } \\
\text { payoff }\end{array}$} & $\begin{array}{l}\text { High quality gap } \\
\theta^{H}>\theta^{L}\left(\frac{1-\mu \lambda}{\mu-\mu \lambda}\right)\end{array}$ & $\begin{array}{l}\text { Small quality gap } \\
\theta^{L}<\theta^{H}<\theta^{L}\left(\frac{1-\mu \lambda}{\mu-\mu \lambda}\right)\end{array}$ & \\
\hline$R_{P A}>R_{P T}$ & $\delta_{A}>\frac{\mu\left(\theta^{H}-\theta^{L}\right)+\theta^{L}}{\mu \lambda\left(\theta^{H}-\theta^{L}\right)+\theta^{L}}+\delta_{T} \frac{\theta^{L}}{\theta^{L}+\mu \lambda\left(\theta^{H}-\theta^{L}\right)}$ & $\delta_{A}>\frac{\mu\left(\theta^{H}-\theta^{L}\right)+\theta^{L}}{\mu \lambda\left(\theta^{H}-\theta^{L}\right)+\theta^{L}}+\delta_{T} \frac{\theta^{L}}{\theta^{L}+\mu \lambda\left(\theta^{H}-\theta^{L}\right)}$ & $\left(B B^{\prime}\right)$ \\
\hline$R_{S e p}>R_{P T}$ & $\delta_{A}>1+\delta_{T}\left[1-\lambda\left(1-\frac{\theta^{L}}{\theta^{H}}\right)\right]$ & $\delta_{A}>1+\delta_{T}\left[1-\lambda\left(1-\frac{\theta^{L}}{\theta^{H}}\right)\right]$ & $\left(A A^{\prime}\right)$ \\
\hline$R_{S e p}>R_{P A}$ & always true & $\delta_{A}<\frac{(1-\mu) \theta^{L}}{\left(\mu \lambda\left(\theta^{H}-\theta^{L}\right)-\mu \theta^{H}+\theta^{L}\right)}+\delta_{T}$ & $\left(C C^{\prime}\right)$ \\
\hline \multicolumn{2}{|l|}{ Ordinates and coefficients } & $1<\frac{\mu\left(\theta^{H}-\theta^{L}\right)+\theta^{L}}{\mu \lambda\left(\theta^{H}-\theta^{L}\right)+\theta^{L}}<\frac{(1-\mu) \theta^{L}}{\left(\mu \lambda\left(\theta^{H}-\theta^{L}\right)-\mu \theta^{H}+\theta^{L}\right)}$ & \\
\hline & $1<\frac{\mu\left(\theta^{H}-\theta^{L}\right)+\theta^{L}}{\mu \lambda\left(\theta^{H}-\theta^{L}\right)+\theta^{L}}$ & $1-\lambda\left(1-\frac{\theta^{L}}{\theta^{H}}\right)<\frac{\theta^{L}}{\theta^{L}+\mu \lambda\left(\theta^{H}-\theta^{L}\right)}$ & \\
\hline & $1-\lambda\left(1-\frac{\theta^{L}}{\theta^{H}}\right)>\frac{\theta^{L}}{\theta^{L}+\mu \lambda\left(\theta^{H}-\theta^{L}\right)}$ & $1-\lambda(i) n$ \\
\hline
\end{tabular}

Table 2. Payoff dominance conditions

Figures 3 and 4 present a map of the equilibria that outline regions where one equilibrium dominates the two other equilibria in terms of payoffs. This equilibrium brings the highest payoff as compared with the other relevant equilibria and should be the preferred choice by the MPH. To obtain this equilibrium the MPH must chose the submission fee that supports it. For instance, if the highest payoff is brought by the Separating equilibrium, the MPH will set $c=c_{1}$.

The "high quality gap" case is presented in Figure 3. Because $\delta_{A}>\delta_{T}$, the area below the $45^{\circ}$ line is not relevant. The Separating equilibrium is always to be preferred to the Pooling Equilibrium A (the frontier separating the the two regions is below the $45^{\circ}$ line). The AA' line delineates the frontier between the Separating and the Pooling Equilibrium T. Above this line, the separating equilibrium dominates both pooling equilibria. Below this line, we know that the Pooling Equilibrium $\mathrm{T}$ is to be preferred to the Separating Equilibrium. A quick look to the BB' line shows that the Pooling Equilibrium T should also be preferred to the Pooling Equilibrium A. So, in the OAA' area, the preferred equilibrium is the Pooling Equilibrium T. Above the AA' frontier, the preferred equilibrium is the separating one. ${ }^{11}$

For relatively small values of $\delta_{T}$, if the accessibility gap between $\delta_{T}$ and $\delta_{A}$ is relatively narrow,

11 The slope of BB' is lower than the slope of AA'. It can be checked analytically that they cannot cross above the $45^{\circ}$ line (which would imply a logical contradiction, where $R P A>R P T>R S E P>R P A$ ). 


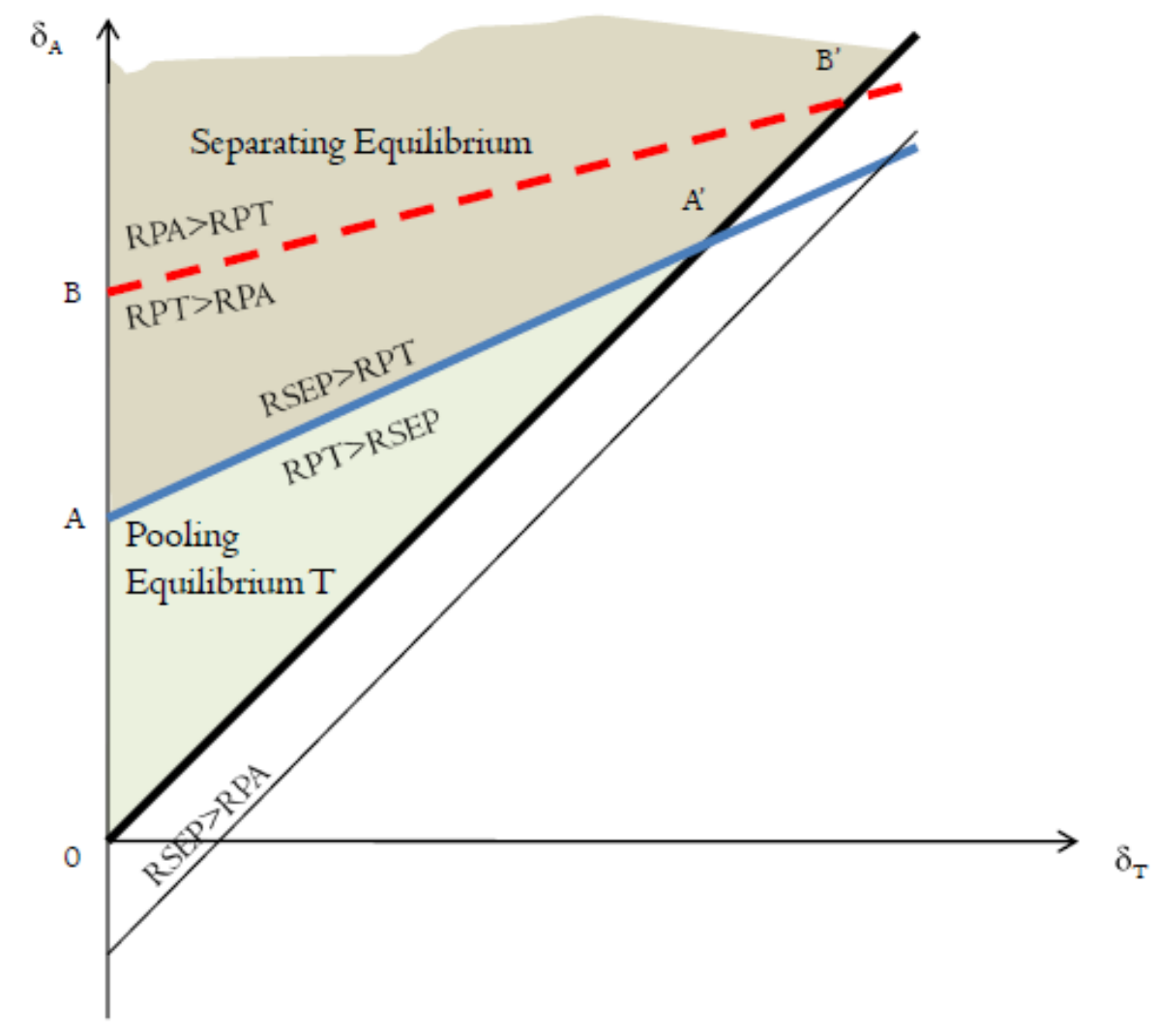

Figure 3: Equilibrium map: The high quality gap case

the MPH would prefer an equilibrium where all authors follow the traditional track, and they keep the "historical" subscription fee. If the gap widens, it might become interesting for the journal to seek the Separating equilibrium. If $\delta_{T}$ is large enough, the Separating equilibrium would be preferred whatever the accessibility gap.

In the same way, Figure 4 analyzes the "low quality gap case"; in this configuration, for a relatively low $\delta_{T}$, a high accessibility gap supports the Pooling Equilibrium A (above the CC' line); an intermediate accessibility gap entails the Separating equilibrium, and, for a low accessibility gap, we get the Pooling Equilibrium T. If $\delta_{T}$ is large enough, the Pooling Equilibrium T is no longer possible.

Thus far, we have considered that the MPH is managing one representative journal. In reality journals cover a different content and target a different readership. If MPHs tune the OA fee to 


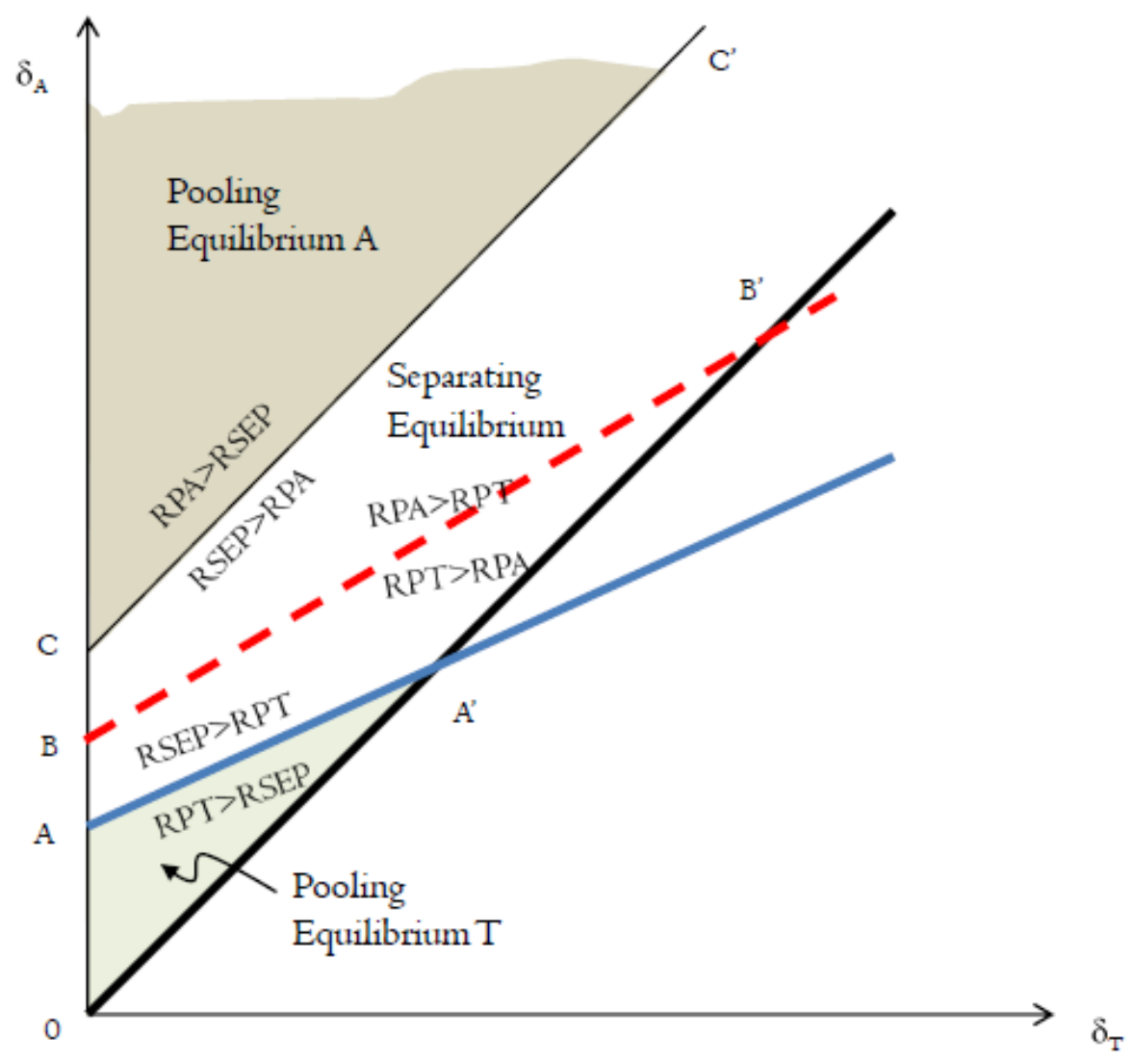

Figure 4: Equilibrium map: the low quality gap case

maximize the income per journal, their publication patterns may evolve toward different equilibria depending on the specific parameters of each journal. Our analysis can help in understanding the future change in journal structures.

For instance, if we agree that "leading journals", i.e. journals unanimously recognized as the best in a given field, have both a low quality gap because of the high standards of the editorial screening process, and a low accessibility gap because experts interested in these journals have easy access to the traditional track (Gaulé and Maystre, 2011), then our analysis suggests that these journals will evolve towards the Separating equilibrium (Figure $4, \delta_{T}$ large, and $\delta_{A}$ close to $\left.\delta_{A}\right)$.

Specialized expert journals also have a low quality gap (Figure 4), but not all libraries have a subscription; thus their open access track should improve paper accessibility in a significant way. 
With a large accessibility gap, these journals would either move toward the Separating equilibrium, or, for a very strong accessibility gap, toward the Pooling equilibrium A.

Finally, second-tier journals are characterized by a high quality gap (Figure 3) and a high accessibility gap, for a relatively small $\delta_{T}$; these would move toward the Separating equilibrium (or the Pooling Equilibrium T).

Table 3 summarizes these scenarios, which are not exhaustive of the whole range of journal profiles.

\begin{tabular}{|l|l|l|l|l|}
\hline Journal types & $\delta_{T}$ & $\begin{array}{c}\text { accessibility gap } \\
\left(\delta_{A}-\delta_{T}\right)\end{array}$ & $\begin{array}{c}\text { quality gap } \\
\left(\theta^{H}-\theta^{H}\right)\end{array}$ & MPH preferred equilibrium \\
\hline leading & high & low & low (Fig. 4) & Separating Equilibrium \\
\hline specialized (good) & high & high & low (Fig. 4) & Separating Eq.; Pooling Eq. A \\
\hline second-tier & low & high & high (Fig. 3) & Separating Eq.; Pooling Eq. T \\
\hline
\end{tabular}

Table 3. Journal types and preferred equilibria

Considering these scenarios, it is quite reasonable to expect that a large number of academic journals will move away from the traditional economic model toward a hybrid form (separating equilibrium) or genuine open-access. In these two cases, the income of the MPH will increase, as will increase the accessibly of scholarly publishing, with an overall positive impact on knowledge dissemination.

\section{Conclusion and welfare implications}

In April 2013 all of the major publishing houses moved thousands of academic journal titles away from the traditional economic model to a hybrid economic model, allowing for an expensive OAper-article option. This paper aims to analyze the consequences of the rise of the hybrid economic model on scholarly publishing. Empirical studies that analyzed the impact of OA facilities as provided by the "old" traditional journals on citations have already revealed that high quality papers tend to self-select for the open access model. Taking stock of this empirical finding, this paper investigates under which conditions authors can use the publication strategy as a signaling 
device.

In our model, because paper accessibility is stronger in the OA track than in the traditional track, authors of high quality papers would pay the high OA fee only to signal their type. Whether authors of low quality papers follow them or not, depends on readers' beliefs and the publication fee as decided by the MPH. If many authors of standard quality papers copy the strategy of the high quality paper authors, the incentive of the latter group to choose the OA track declines. The MPH uses the publication fee as an equilibrium selection device tuning it to maximize its income. We solved the game for the various equilibria and determined the optimal publication fee in each equilibrium. The relevant equilibria include a Separating equilibrium - where authors of high quality papers self-select to the OA track and authors of low-quality papers self-select for the traditional track - and two Pooling equilibria, where all authors choose one of the two pure strategies (either OA or traditional). Finally, we present an equilibrium map depending on the quality gap and accessibility gap. This allows us to anticipate the possible evolution of a journal structure, depending on its profile.

One important finding of this analysis is that, with the exception of second-tier journals, many homogenous quality journals would have an incentive to move toward the hybrid model; and perhaps even toward the more extreme full open access structure. This move will come with higher revenues for the MPHs, a reduced burden for libraries, and high costs for authors.

Raising income for the MPHs and off-setting raising publication costs for authors and their host Institutions is the mark of the excessive market power of MPHs; this trend can be interpreted as a negative for knowledge dissemination. Nonetheless, the fact that leading journals might extend their OA track can be seen as good news for knowledge dissemination, given the improved access for scholars working in poorer regions.

Furthermore, the emergence of the Separating equilibrium where high quality papers will select for the OA track can also contribute to increase the overall efficiency of research, given that readers of journals evolving in this direction will now have a powerful tool to detect quality, and can avoid investing time and effort in reading low quality papers. 


\section{References}

Bowen, Elizabeth A., Mark A. Mattaini and Sandra L. De Groote, 2013. Open access for social work research and practice, Journal of the Society for Social Work and Research 4 (1): $31-46$.

Besancenot, Damien, Kim V. Huynh, and Joao R. Faria, 2012. Search and research: the influence of editorial boards on journals' quality, Theory and Decision 73 (4): 687-702.

Besancenot, Damien, and Radu Vranceanu, 2008. Can incentives for research harm research? A business schools' tale. Journal of Socio-Economics 37 (3): 1248-1265.

Dewatripont, Mathias, Ginsburgh, Victor, Legros, Patrick, and Alexis Walckiers, 2007. Pricing of scientific journals and market power, Journal of the European Economic Association $5(2-3): 400-410$.

Edlin, Aaron S., and Daniel L. Rubinfeld, 2005. The bundling of academic journals. American Economic Review 95 (2): 441-446.

Gaulé, Patrick and Nicolas Maystre, 2011. Getting cited: does open access help?, Research Policy 40 (10): 1332-1338.

Harnad, Steven and Tim Brody. 2004. Comparing the impact of Open Access (OA) vs. non-OA articles in the same journals, D-Lib Magazine 10 (6).

Jeon, Doh-Shin and Jean-Charles Rochet, 2010. The pricing of academic journals: A twosided market perspective, American Economic Journal: Microeconomics 2 (2): 222-255.

Kurtz, Michael J., Eichhorn, Guenther, Accomazzi, Alberto, Grant, Carolyn, Demleitner, M., Henneken, E. and Stephen S. Murray, 2005. The effect of use and access on citations, Information Processing and Management 41 (6): 1395-1402.

Lawrence, Steve, 2001, Free online availability substantially increases a paper's impact, Nature 411: 521.

McCabe, Mark J., and Christopher M. Snyder, 2013b. Identifying the effect of open access on citations using a panel of science journals, mimeo, University of Michigan, http://mccabe.people.si.umich.edu/McCabe_Snyder_EI_2013.pdf.

McCabe, Mark J., and Christopher M. Snyder, 2013a. Does online availability increase citations? Theory and evidence from a panel of economics and business journals, Review of Economics and Statistics, In print.

McCabe, Mark J., and Christopher M. Snyder, 2007. Academic journal prices in a digital age: A two-sided market model, BE Journal of Economic Analysis and Policy 7.(1).

McCabe, Mark J., and Christopher M. Snyder, 2005. Open access and academic journal quality, American Economic Review 95 (2): 453-459.

Nevo, Aviv, Daniel L. Rubinfeld, and Mark McCabe, 2005, Academic journal pricing and the demand of libraries, American Economic Review 95 (2): 447-452.

Spence, Michael, 1973. Job market signaling, Quarterly Journal of Economics 87 (3): 355374 .

Walker, Thomas, 2004. Open access by the article: an idea whose time has come? Nature Web Focus, 15 April, 1004, http://www.nature.com/nature/focus/accessdebate/13.html. 
ESSEC Business School Avenue Bernard Hirsch BP 50105

95021 Cergy-Pontoise Cedex France

Tél. +33(0)134433000

$\mathrm{Fax}+33(0) 134433001$

www.essec.fr

\section{ESSEC Executive Education} CNIT BP 230

92053 Paris-La Défense France

Têl. + $33(0) 146924900$

Fax +33 (0)1 46924990

http://formation.essec.fi

ESSEC Business School

Singapore Campus

100 Victoria Street

National Library Building \# 13-02

Singapore 188064

essecasia@essec.fr

Tél. +6568849780

Fax +6568849781

www.essec.edu

Informations

Alison Bougi

+33 (0)134433358

bougi@essec.fr

www.essec.fr

research.center@essec.fr

ISSN 1291-9616 